\title{
Estudo da emissão de raios infravermelho próximo em processos de soldagem a arco
}

\author{
(Study of near-infrared emission on processes of arc welding)
}

\begin{abstract}
Carolina Pimenta Mota ${ }^{1}$, Marcus Vinícius Ribeiro Machado ${ }^{1}$, Diandro Bailoni Fernandes ${ }^{1}$, Louriel Oliveira Vilarinho ${ }^{1}$ ${ }^{1}$ Universidade Federal de Uberlândia, Faculdade de Engenharia Mecânica, Uberlândia, Minas Gerais, Brasil, cpimentamota@gmail. com,mvrmachado@gmail.com,diandro@mecanica.ufu.br, vilarinho@mecanica.ufu.br
\end{abstract}

\begin{abstract}
Resumo
O estudo de boa parte dos fenômenos envolvidos no processo de soldagem necessita de auxílio visual e a luminosidade emitida pelo arco pode representar uma grande barreira. Uma das formas utilizadas atualmente para se obter a visualização do processo, sem a interferência do arco, consiste em iluminar o processo com o infravermelho próximo e utilizar filtros durante a aquisição das imagens. Assim, é importante investigar o comportamento do arco de soldagem em relação à sua emissão luminosa no espectro infravermelho. Desta forma, a proposta deste trabalho foi a realização de um estudo comparativo entre a emissão do arco de soldagem de radiação infravermelha próxima em dois processos largamente utilizados, TIG e MIG/MAG, focando também sua influência por parâmetros como a corrente de soldagem e a proteção gasosa utilizada. Com o uso de um sensor de luminosidade e a utilização de um sistema de lentes ópticas, foram realizados experimentos, adquirindo o espectro luminoso emitido pelo arco voltaico. Através dos resultados obtidos, ou seja, do valor numérico de energia luminosa do arco de soldagem (integração do espectro) é possível se obter, com a utilização desta mesma metodologia, a energia luminosa no infravermelho próximo e, consequentemente, a intensidade luminosa, necessária para a sobreposição do arco durante a aquisição de imagens.
\end{abstract}

Palavras-chave: Soldagem, Radiação infravermelha próxima; Auxílio Visual

\begin{abstract}
Most of the phenomenon studied in the welding processes needs a vision system and the arc light emission can create a great barrier. Nowadays, one of the techniques used for visualizing the process, without arc interference, is the illumination of the process with near-infrared laser and the use of optic filters during the image acquisition. Thus, it is important to investigate the welding arc behavior in respect to its light emission within the near-infrared spectrum. Therefore, this work aims to perform a comparative study of the arc near infrared emission in one of the two most used processes: TIG and MIG/MAG, focusing on the influence of welding parameters, such as current and shielding gas. Through a luminous sensor and the use of an optic lens system, experiments were carried out by acquiring the light spectra emitted by the welding arc. It's possible, using the arc results - arc energy value - obtained and following the same methodology proposed in this work, to know the quantity of infrared energy, and its luminosity, needed to overlay the arc during the image acquisition.
\end{abstract}

Key-words: Welding; Near Infrared radiation; Vision System.

\section{Introdução}

A soldagem é um dos principais processos de fabricação utilizados na indústria para a união de materiais, principalmente os metais, sendo cada vez mais necessário e mais empregado. Tendo isso em vista, a necessidade da garantia de qualidade e produtividade dos processos envolvidos nesta tarefa está em constante crescimento. Sendo um processo especial, com parâmetros interdependentes, muitos trabalhos de pesquisa são desenvolvidos com o intuito de alcançar um melhor entendimento de seus fenômenos envolvidos.

(Recebido em 04/10/2010; Texto final 17/03/2011).

Artigo originalmente publicado no CONSOLDA 2009,
Uma das formas amplamente aplicadas, atualmente, para o auxílio dos estudos do processo de soldagem são os sistemas de visão. O uso de câmeras, de baixa ou alta velocidade, pode fornecer diversas informações úteis aos pesquisadores, que não são possíveis de se obter com a monitoração dos sinais elétricos usuais, como a corrente ou a tensão. Diferentes sistemas de visão podem fornecer informações específicas sobre o processo em andamento: a técnica de perfilografia ou back-lighting - projeção da sombra de elementos na região da solda em uma superfície plana - ilustrada na Figura 1, visualiza as gotas em transferência como uma forma de estudo da transferência metálica Bálsamo et al. [1], enquanto novos sistemas de visão têm como o objetivo a visualização direta do metal fundido e da junta propriamente dita, com uma gama de aplicações indo de estudo dos fenômenos na poça até seguidores de junta ou controladores online de parâmetros do processo. 


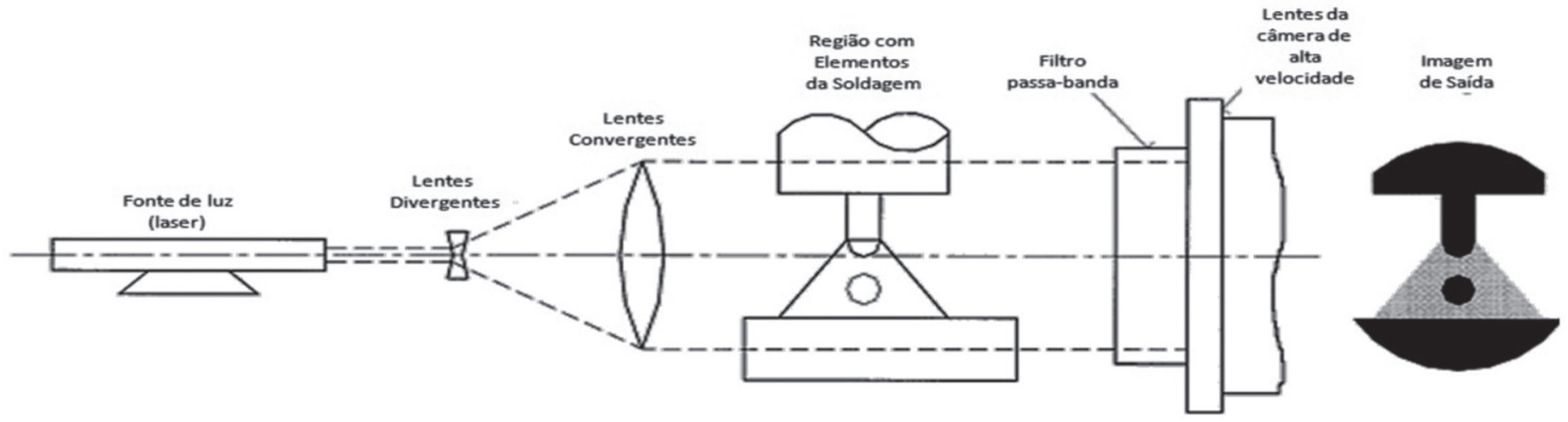

Figura 1. Visualização da transferência metálica por perfilografia de Bálsamo et al. [1].

A visualização da poça fundida pode dar informações sobre muitos aspectos da solda. Diversos estudos, como Zhang et al. [2], Zhang et al. [3], Baskoro et al. [4], Vilarinho et al. [5], comprovam que a observação e o controle da poça fundida, e suas principais características, resulta na penetração e propriedades mecânicas desejadas, enquanto Hara and Suga [6] propuseram um sistema de controle de penetração pela frequência natural e largura da poça fundida. Além disso, a aplicação de um sistema de auxílio visual vai muito além do controle da penetração em um processo de soldagem. Uma visualização nítida da superfície da poça fundida e sua deformação, segundo Duffey et al. [7], retêm informações importantes de vários fenômenos básicos, como o fluxo de Marangoni dentro da poça de soldagem, o recuo da pressão devido à vaporização metálica, entre outros.

$\mathrm{O}$ arco voltaico nos processos de soldagem emite, além da radiação luminosa visível, em praticamente todos os comprimentos de onda, incluindo radiações ultravioleta e infravermelha. A intensidade luminosa emitida, entretanto, em comprimentos de onda infravermelhos superiores a $850 \mathrm{~nm}$ é pequena quando comparada ao espectro visível, Figura 2. Uma das técnicas de visualização do processo é a utilização de filtros passa longa nesta região de emissão, com o intuito de diminuir a luminosidade do arco presente durante a aquisição de imagens. Esta técnica, apesar de eficiente, não é suficiente para "apagar" completamente o arco, Figura 3, e a luminosidade restante afeta a precisão e a nitidez com que se observa a poça fundida, não sendo suficiente em aplicações que demandem maior precisão.

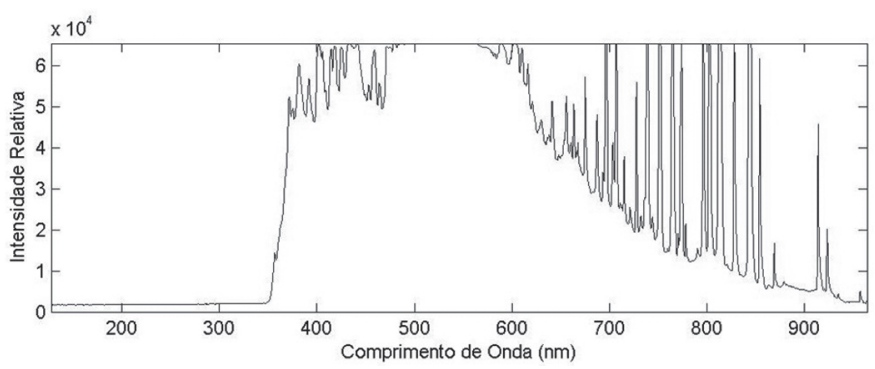

Figura 2. Exemplo de espectro do arco (TIG, Ar puro, 150A).

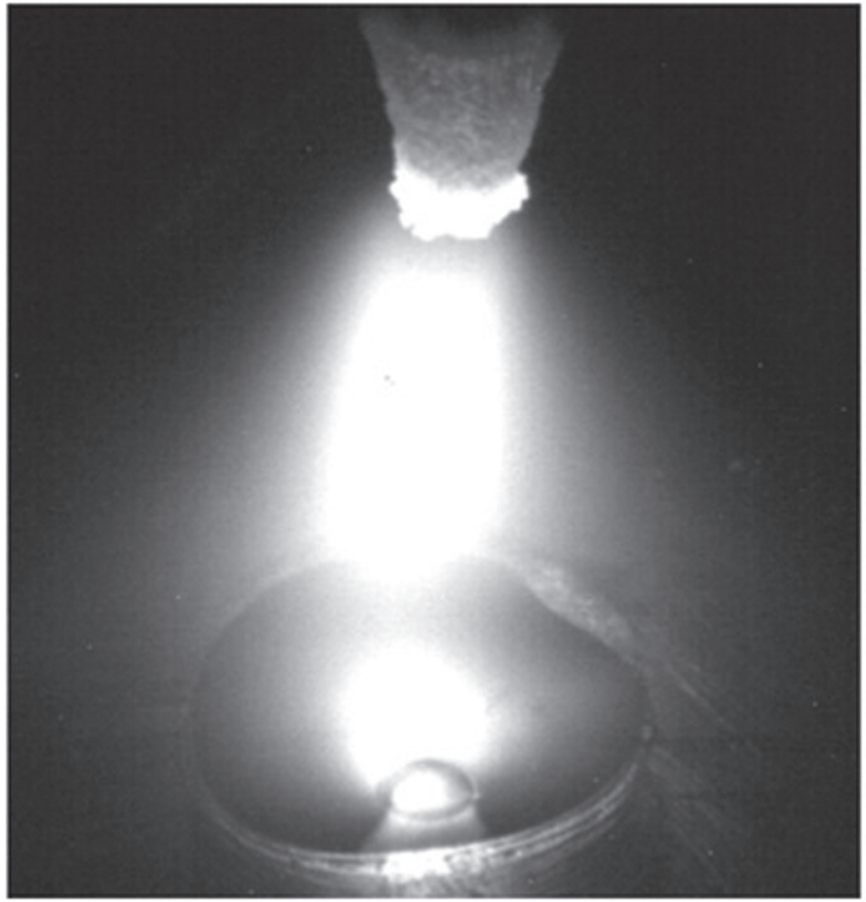

Figura 3. TIG em aço carbono, Ar puro, 100A, com filtro óptico passa longa de $850 \mathrm{~nm}$ de Vilarinho et al. [5].

Para que a luminosidade do arco não seja um problema durante a aquisição de imagens, utiliza-se a técnica de Iluminação a Laser com Filtragem de Espectro. Segundo Houghton et al. [8], o princípio da técnica é diminuir ou eliminar totalmente a luz do arco que alcança a câmera iluminando a área de solda com a luz de um laser. A fim de minimizar os requerimentos de intensidade da iluminação, o comprimento de onda da luz da fonte de iluminação deve ser selecionado onde a intensidade do arco é baixa. Um filtro passa banda estreito é necessário para eliminar a luz do arco e apenas a luz do laser será capaz de alcançar a câmera, como mostra a Figura 4.

Neste contexto, é importante o conhecimento do comportamento do arco em relação à sua emissão luminosa, principalmente a infravermelha. Desta forma, a proposta deste trabalho foi a realização de um estudo comparativo entre a 


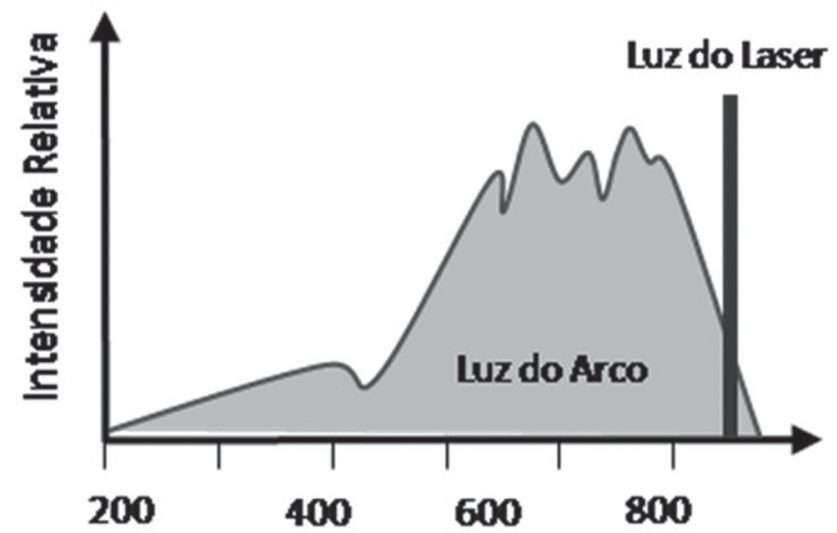

Comprimento de onda, $\mathrm{nm}$

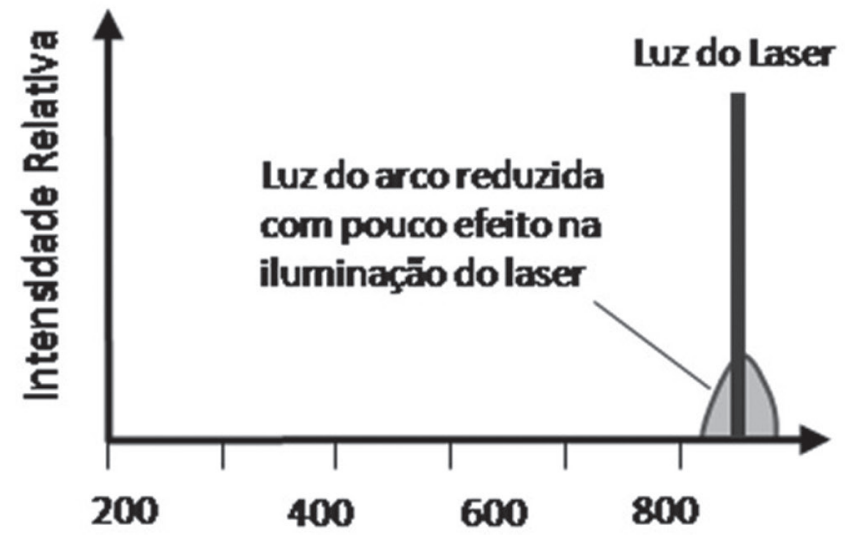

Comprimento de onda, $\mathrm{nm}$

Figura 4. Princípio da Filtragem do Espectro segundo Houghton et al. [8].

emissão do arco de soldagem de infravermelho próximo (neste caso, nas proximidades de $905 \mathrm{~nm}$ ) nos processos TIG (GTAW) e MIG/MAG (GMAW), avaliando também sua influência pela intensidade de corrente e composição da proteção gasosa empregada.

\section{Metodologia Experimental}

A fim de atingir os objetivos deste trabalho, elaborou-se uma metodologia padrão de trabalho, envolvendo desde a escolha dos parâmetros de soldagem dos dois processos distintos, passando pela fabricação e ajuste do sensor de luminosidade até o desenvolvimento de um programa para a obtenção e a interpretação do espectro do arco de solda. A padronização de todas as etapas envolvidas neste experimento possibilitou a comparação e discussão dos resultados obtidos.

Como sensor de luminosidade, para a obtenção do espectro do arco, utilizou-se de um Espectrômetro portátil, modelo USB, com resolução de comprimento de onda de $0,3 \mathrm{~nm}$, entrada de fibra óptica SMA905, detector CCD de 2048 pixels. Além de sua portabilidade e versatilidade (uso de uma fibra com maior resolução ou maior captação de luz), sua comunicação por USB facilita a sua interface com plataformas de programação, como o LabView ${ }^{\circledR}$, deixando-o independente de seu software original. A fibra ótica utilizada, com diâmetro de 600 micrômetros e comprimento de 2 metros, capta a radiação e a conduz até o espectrômetro.

Para a focalização do ponto da emissão luminosa, utilizouse de um suporte com um sistema de lentes convergentes e divergentes, Figura 5, e um filtro óptico passa banda em 905 $\mathrm{nm}$, comprimento de onda de interesse para desenvolvimentos futuros do sistema de visão. Calcularam-se, por sim, as distâncias necessárias entre as lentes para que o arco focalizado pudesse ser captado inteiramente e enviado para a fibra ótica, como ilustra a Figura 6. É importante ressaltar que a lente divergente (com comprimento focal de $-12 \mathrm{~mm}$ ) e a lente convergente subsequente (com comprimento focal de $54 \mathrm{~mm}$ ) estão alinhadas com seu foco coincidente e a fibra ótica está nas proximidades do ponto focal da última lente convergente (com comprimento focal de $54 \mathrm{~mm})$. A distância entre o orifício delimitador $(\varnothing=10$ $\mathrm{mm}$ ) e o arco de solda (D) determina o tamanho do objeto visto pelo espectrômetro.

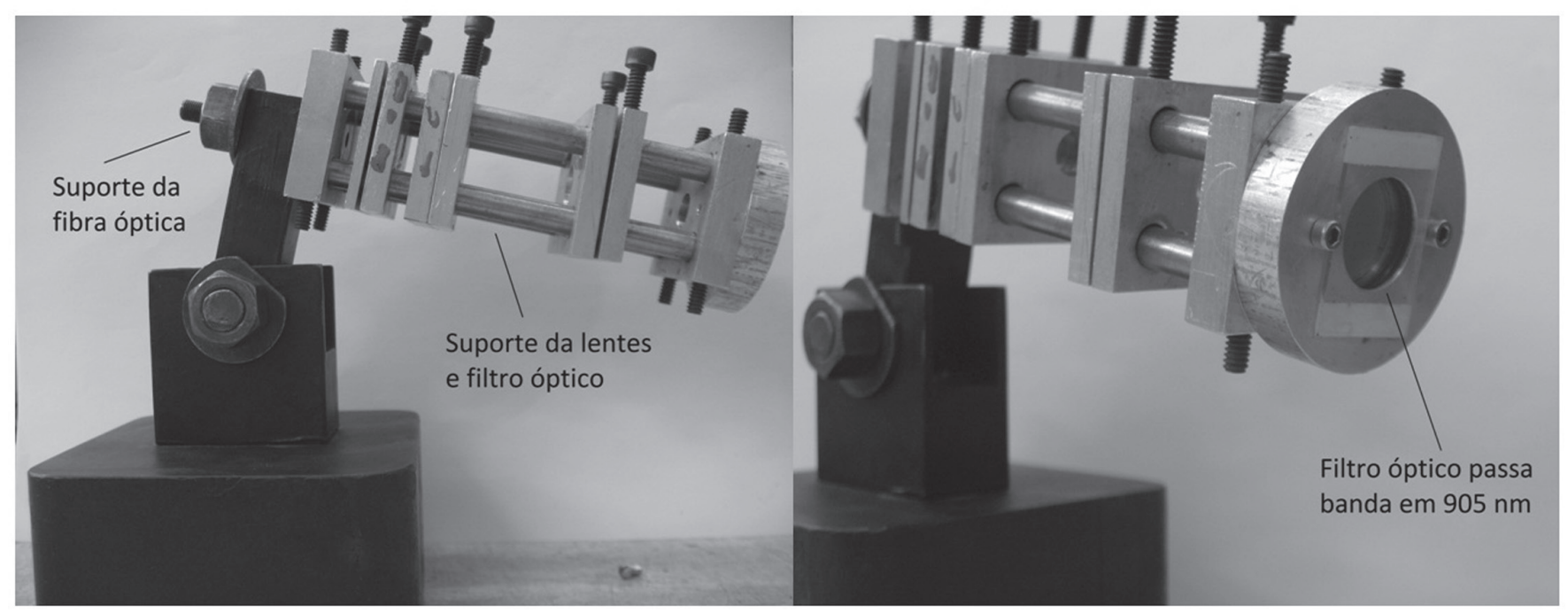

Figura 5. Suporte para fibra óptica e lentes (esquerda) e detalhe para filtro óptico passa banda (direita). 
Todos os testes foram realizados com o auxílio de uma tocha acoplada a um braço robótico, sem movimento em relação ao espectrômetro. O suporte da fibra óptica e das lentes foi posicionado de modo que apenas uma região envolvendo a placa de teste e a tocha de soldagem fosse focalizada pelo sistema de lentes, sem que ocorresse saturação completa do sensor, com uma distância em linha reta entre eles (D) de $795 \mathrm{~mm}$. As chapas de teste foram posicionadas sobre uma mesa com controle de velocidade de movimentação e a solda realizada em direção ao suporte (mesa movimentando na direção contrária), para evitar a interposição da tocha entre o arco e a poça fundida com o sensor, Figura 7.

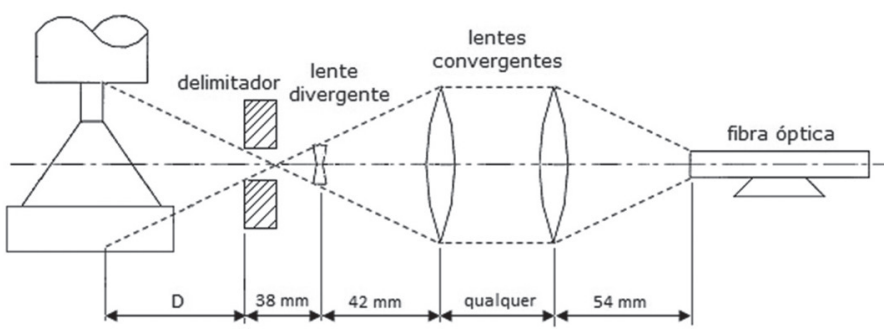

Figura 6. Esquema do sistema de lentes para focalização do arco na fibra óptica.

O espectro e a radiação do arco foram adquiridos através de um programa em ambiente LabView ${ }^{\circledR}$, Figura 8, com a calibração e bibliotecas fornecidas pelo próprio fabricante do espectrômetro. $\mathrm{O}$ usuário deve entrar com quatro dados para a aquisição durante os experimentos: o tempo de integração, tempo que o sensor CCD do espectrômetro permanece aberto para a obtenção de um sinal; o número de médias, quantidade de vezes que o sensor faz o cálculo durante um ciclo; o intervalo de tempo entre duas amostras e o número de amostras de espectro a serem gravadas. Neste trabalho foram utilizados os valores de $40 \mathrm{~ms}$, 5, $200 \mathrm{~ms}$ e 10, respectivamente. Ao clicar no botão "Gravar", o programa salva automaticamente em arquivos LVM sucessivos e no intervalo definido o vetor de intensidades luminosas.

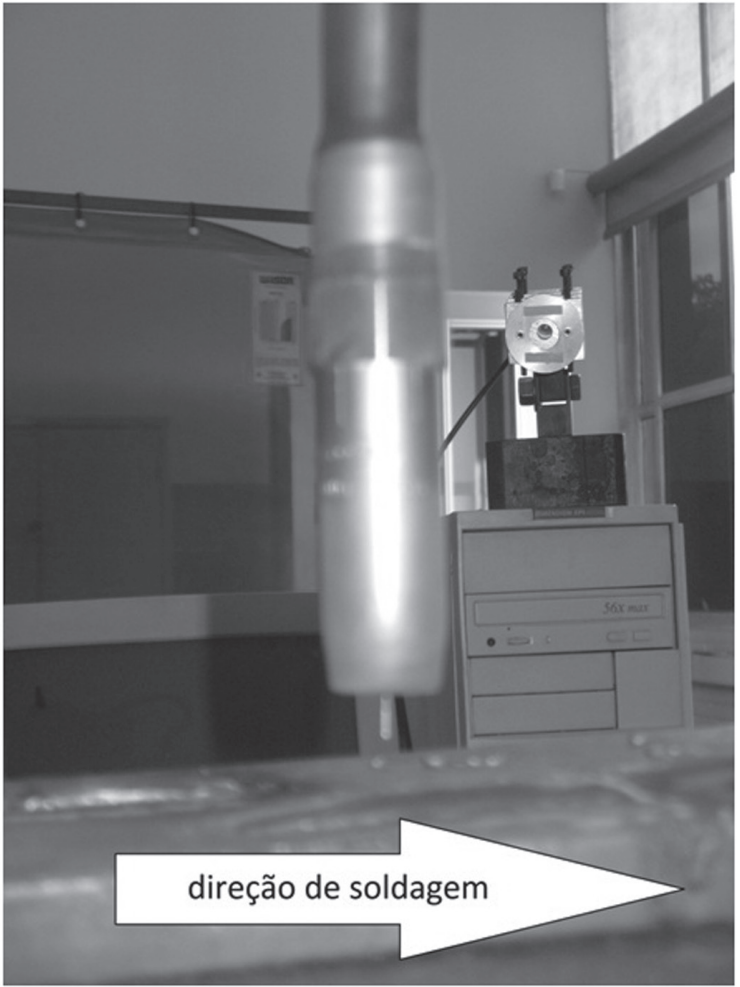

Figura 7. Posicionamento da tocha e suporte do espectrômetro.

A tabela mostrada a seguir, Tabela 1, relaciona os parâmetros de soldagem utilizados nos testes para a aquisição do espectro do arco. Utilizando-se de dois processos a arco, TIG e MIG/MAG, variou-se a proteção gasosa e a intensidade de corrente. Ainda, em cada processo, ajustaram-se os demais parâmetros para obter uma transferência metálica do tipo spray (não há grande variação na emissão de radiação ao longo do tempo) e um comprimento de arco aproximadamente similar entre os testes. Por fim, todos os testes foram realizados com o uso do filtro passa banda em $905 \mathrm{~nm}$, exceto um teste para cada processo com a finalidade de comparação entre os espectros.

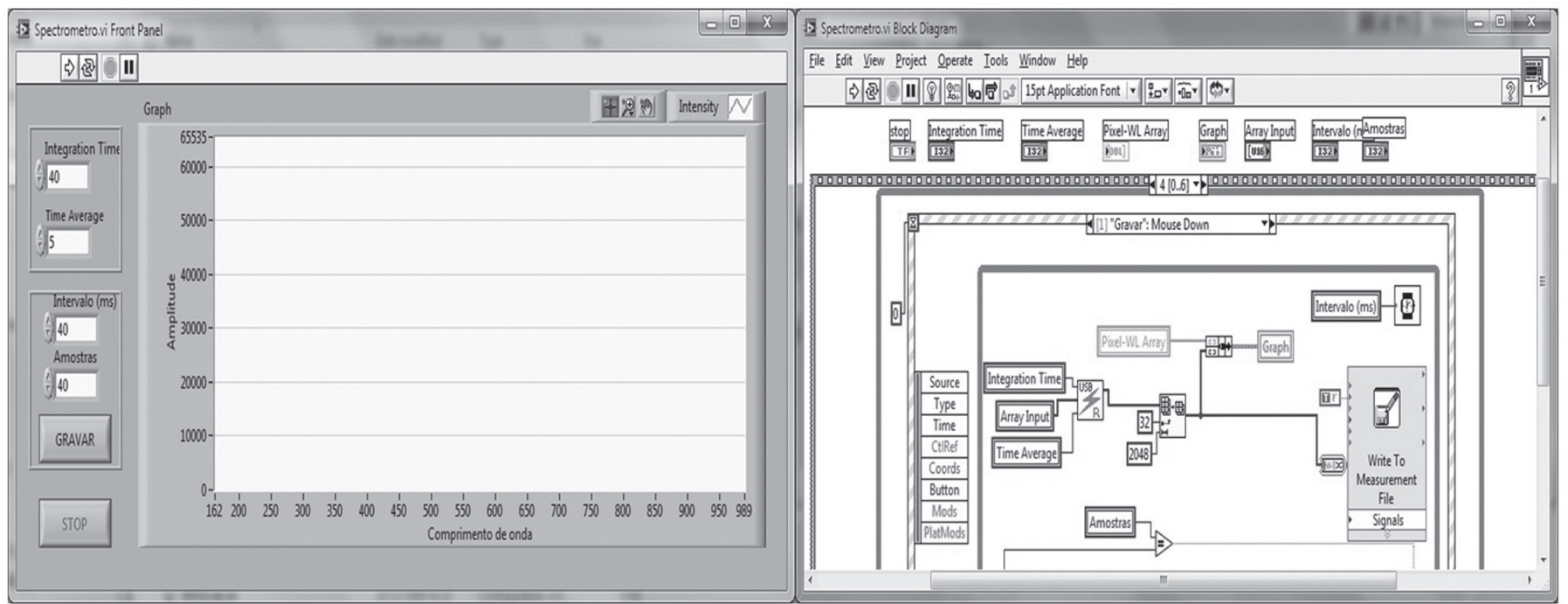

Figura 8. Telas do programa em LabView ${ }^{\circledR}$ para aquisição do espectros de luz: (esquerda) tela de trabalho e interface com o usuário e (direita) modo de edição do programa. 
Tabela 1. Parâmetros utilizados para a realização dos experi

\begin{tabular}{|c|c|c|c|c|c|c|c|c|}
\hline Teste & Processo & $\begin{array}{c}\text { Corrente } \\
\text { (A) }\end{array}$ & $\begin{array}{l}\text { Vel. de Alim. } \\
\quad(\mathrm{m} / \mathrm{min})\end{array}$ & $\begin{array}{l}\text { Vel. Soldagem } \\
(\mathrm{cm} / \mathrm{min})\end{array}$ & $\begin{array}{c}\text { Proteção } \\
\text { Gasosa }\end{array}$ & $\begin{array}{l}\text { Vazão } \\
\text { (l/min) }\end{array}$ & $\begin{array}{c}D B C P / D E P^{(l)} \\
(\mathrm{mm})\end{array}$ & $\begin{array}{l}\text { Filtro } \\
905 \mathrm{~nm}\end{array}$ \\
\hline 1 & TIG & 150 & - & 20 & Argônio & 13 & 4 & SEM \\
\hline 2 & TIG & 150 & - & 20 & Argônio & 13 & 4 & $\mathrm{COM}$ \\
\hline 3 & TIG & 200 & - & 20 & Argônio & 13 & 4 & $\mathrm{COM}$ \\
\hline 4 & TIG & 250 & - & 20 & Argônio & 13 & 4 & $\mathrm{COM}$ \\
\hline 5 & MIG/MAG & 200 & $19^{(2)}$ & 25 & Argônio & 15 & 22 & $\mathrm{COM}$ \\
\hline 6 & MIG/MAG & 250 & $22^{(2)}$ & 25 & Argônio & 15 & 18 & $\mathrm{COM}$ \\
\hline 7 & MIG/MAG & 275 & 7,8 & 25 & Argônio & 15 & 22 & SEM \\
\hline 8 & MIG/MAG & 275 & 7,8 & 25 & Argônio & 15 & 22 & $\mathrm{COM}$ \\
\hline 9 & MIG/MAG & 390 & 12,5 & 35 & Argônio & 15 & 22 & $\mathrm{COM}$ \\
\hline 10 & MIG/MAG & 275 & 7,8 & 25 & $\mathrm{Ar}+8 \% \mathrm{CO}_{2}$ & 15 & 22 & $\mathrm{COM}$ \\
\hline 11 & MIG/MAG & 390 & 13,2 & 35 & $\mathrm{Ar}+8 \% \mathrm{CO}_{2}$ & 15 & 22 & $\mathrm{COM}$ \\
\hline 12 & MIG/MAG & 275 & 7,8 & 25 & $\mathrm{Ar}+5 \% \mathrm{O}_{2}$ & 15 & 22 & $\mathrm{COM}$ \\
\hline 13 & MIG/MAG & 390 & 13 & 35 & $\mathrm{Ar}+5 \% \mathrm{O}_{2}$ & 15 & 22 & $\mathrm{COM}$ \\
\hline
\end{tabular}

${ }^{(1)}$ Nota 1: DBCP é a distância entre o bico de contato e a peça em soldagens MIG/MAG e DEP é a distância entre o eletrodo e a peça em soldagens $T I G$.

${ }^{(2)}$ Nota 2: os testes de número 5 e 6 foram realizados com arame 0,8 $\mathrm{mm}$.

Segue lista dos equipamentos e consumíveis utilizados durante a realização dos testes:

- Fonte Fronius TransPlus Synergic 5000 para o processo MIG/MAG;

- Arame ER70S6 com diâmetro de $0,8 \mathrm{~mm}$ testes 5 e 6 ;

- Arame ER70S6 com diâmetro de 1,2 mm para demais testes em MIG/MAG,

- Fonte IMC MTE digitec 300 para o processo TIG;

- Eletrodo EWTh-2 com diâmetro de 2,4 mm;

- Gases de proteção: Argônio, $\mathrm{Ar}+8 \% \mathrm{CO}_{2,} \mathrm{Ar}+5 \% \mathrm{O}_{2}$;

- Chapas de aço ao carbono com espessura de 1/2";

- Suporte para lentes com ajuste de distâncias;

- Lentes divergente (-12 $\mathrm{mm}$ ) e convergentes (54 mm);

- Filtro Óptico Comar 905 IH 25;

- Mesa movimentadora IMC SDP-600;

Para a interpretação dos vetores de intensidade luminosa coletados durante o experimento, desenvolveu-se um programa em linguagem MATLAB ${ }^{\circ}$. $O$ algoritmo percorre todas as pastas de arquivos LVM contidos em um determinado diretório, relaciona os vetores de intensidade com um vetor de calibração dos comprimentos de onda, calcula os valores médios para cada ponto de comprimento, imprime os vetores e sua média em um gráfico e calcula a integral numérica do espectro médio obtido, através de um algoritmo de integração numérica pelo método Trapezoidal, descrito por Cheney and Kincaid [9]. Todos os espectros foram subtraídos do valor DC correspondente à emissão nula, para o cálculo da integral e o valor desta será utilizado como fator de comparação entre os testes.
Deve-se ressaltar que os valores obtidos, tantos da intensidade luminosa em cada ponto do espectro adquirido, como o valor da integral numérica calculada são apenas valores relativos, dependentes de inúmeros fatores como o tempo de integração, o número de médias, a posição do espectrômetro em relação à soldagem e as condições do teste. Portanto, os resultados aqui obtidos são úteis apenas quando utilizados em forma de comparação com testes realizados exatamente na forma descrita neste item, não sendo possíveis conclusões fora da abrangência desta metodologia experimental.

\section{Resultados e Discussão}

Os valores da integração numérica, na faixa de comprimentos de 890 e $930 \mathrm{~nm}$, obtidos após a coleta e interpretação dos espectros, as tensões e correntes médias monitoradas durante os processos estão listado na tabela a seguir, Tabela 2. Nos testes de número 1 e 7 o filtro óptico passa banda em $905 \mathrm{~nm}$ foi retirado para a aquisição do espectro completo do arco e foi realizada a integração de toda a faixa de comprimentos. Através da Figura 9, podemos observar que o filtro óptico, além de anular as emissões com comprimento de onda fora da banda passante (a resposta deste filtro é uma gaussiana com $10 \mathrm{~nm}$ de largura de banda), atenua a intensidade do espectro dentro da banda passante.

Deve-se ressaltar que o perfil de intensidades espectral obtido pelo sensor de luminosidade utilizado neste trabalho, assim como seu valor de integração, são relativos, dependentes de todos os aspectos descritos na metodologia experimental. 
Tabela 2. Resultados obtidos nos experimentos.

\begin{tabular}{c|cccccc}
\hline Teste & Processo & Corrente $(A)$ & $\begin{array}{c}\text { Filtro } \\
905 \mathrm{~nm}\end{array}$ & $\begin{array}{c}\text { Corrente } \\
\text { Monitorada }(A)\end{array}$ & $\begin{array}{c}\text { Tensão } \\
\text { Monitorada }(V)\end{array}$ & \begin{tabular}{c} 
Integração \\
\hline 1
\end{tabular} \\
\hline 2 & TIG & 150 & SEM & 151 & 14,7 & $2,28 \mathrm{E}+07$ \\
3 & TIG & 150 & COM & 151 & 15,2 & $4,05 \mathrm{E}+04$ \\
4 & TIG & 200 & COM & 202 & 16,1 & $5,22 \mathrm{E}+04$ \\
5 & TIG & 250 & COM & 253 & 17,8 & $7,90 \mathrm{E}+04$ \\
6 & MIG/MAG & 200 & COM & 202 & 31,5 & $7,15 \mathrm{E}+04$ \\
7 & MIG/MAG & 250 & COM & 250 & 33,5 & $9,74 \mathrm{E}+04$ \\
8 & MIG/MAG & 275 & SEM & 275 & 29,6 & $3,50 \mathrm{E}+07$ \\
9 & MIG/MAG & 275 & COM & 275 & 29,6 & $3,23 \mathrm{E}+05$ \\
10 & MIG/MAG & 390 & COM & 393 & 33,5 & $5,65 \mathrm{E}+05$ \\
11 & MIG/MAG & 275 & COM & 267 & 26,6 & $2,39 \mathrm{E}+05$ \\
12 & MIG/MAG & 390 & COM & 394 & 31,7 & $4,43 \mathrm{E}+05$ \\
13 & MIG/MAG & 275 & COM & 268 & 24 & $1,71 \mathrm{E}+05$ \\
\hline
\end{tabular}
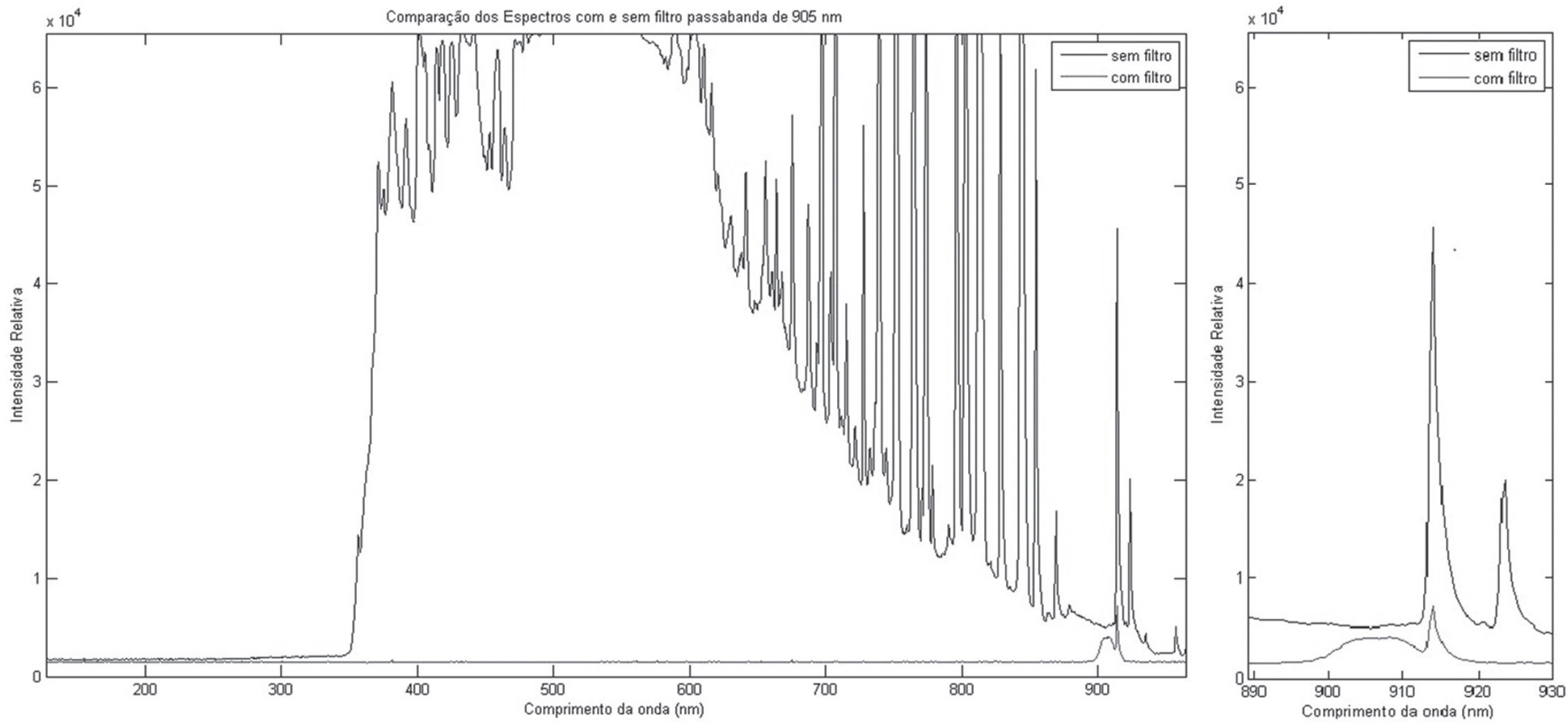

Figura 9. Comparação dos espectros com e sem o uso do filtro (esquerda) e detalhe para atenuação na faixa 890-930 nm (direita), testes 1 e 2 .

Uma segunda análise pode ser discutida através da comparação entre os testes de número 8 a 13, Figura 10. Com o aumento da corrente, mantendo-se o mesmo tipo de proteção gasosa, houve um aumento do valor da integração do espectro, ou seja, uma maior emissão de radiação infravermelha. O aumento da corrente, mantendo-se o mesmo comprimento do arco, entrega maior energia ao arco, aumentando a agitação dos átomos presentes e, portanto, aumentando o número de partículas ionizadas, corroborado por trabalho de Vilarinho [10]. Sabe-se que parte da radiação infravermelha deve-se à emissão de radiação atômica, quando um elétron pertencente a um átomo excitado libera energia luminosa ao retonar à sua posição de origem e, portanto, quanto maior o número de partículas ionizadas, maior será a energia luminosa emitida com o retorno dos elétrons aos seus estados naturais. 


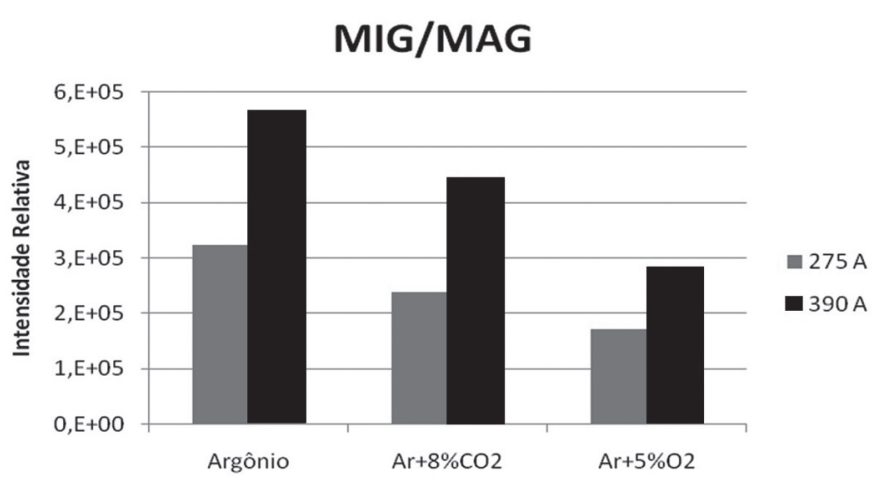

Figura 10. Resultados da integração numérica para testes 7 a 13.

Outra análise interessante retirada destes mesmos testes (8 a 13) é a influência da proteção gasosa sobre emissão de infravermelho. Pode-se observar que, para qualquer corrente, a integração numérica apresentou os maiores valores para a proteção de Argônio puro, valores intermediários com a adição de dióxido de carbono e, por fim, valores menores com a adição de oxigênio ao argônio. Ao comparar os espectros médios obtidos, Figura 11, nota-se a diferença entre os espectros dos gases de proteção, onde o teste com argônio apresentou um grande pico em torno de $914 \mathrm{~nm}$, o teste com adição de dióxido de carbono apresentou picos menores abaixo deste valor e, por fim, o teste com adição de oxigênio apresentou um pico menor em torno dos mesmos $914 \mathrm{~nm}$.

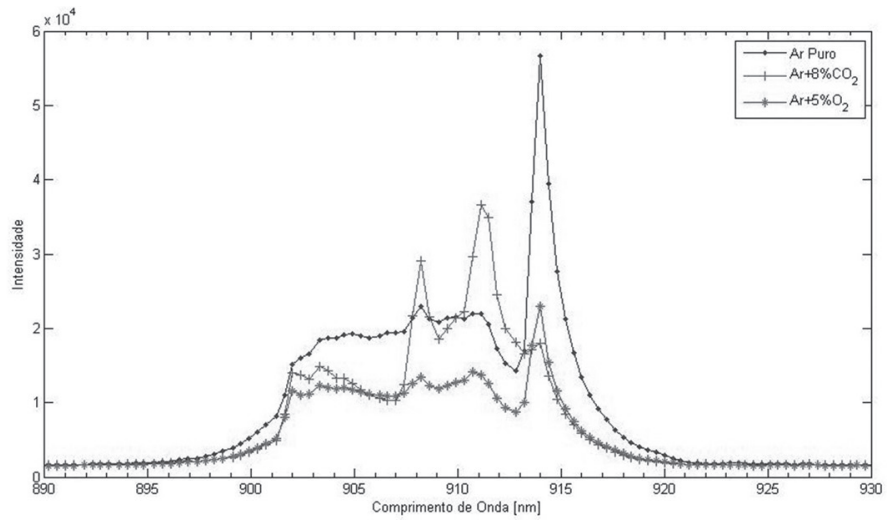

Figura 11. Comparativo espectro dos testes 8, 10 e 12 (275 A).

Sabe-se que o espectro adquirido na região ao redor do arco é composto pela radiação térmica de corpo negro, devido à alta temperatura atingida, e correspondente à área inferior do espectro obtido, e pelo espectro de emissão atômica, quando um elétron pertencente a um átomo excitado libera energia ao retonar à sua posição de origem, e correspondente aos picos presentes. De acordo com National Institute of Standards and Technology (NIST) [11], os átomos excitados presentes nos diferentes gases utilizados (argônio, carbono e oxigênio), possuem picos de emissão na faixa de comprimentos de onda filtrados, como mostra a Tabela 3, e são, portanto responsáveis pelos picos presentes nos espectro adquiridos.
Tabela 3. Picos de emissão dos átomos excitados presentes nos gases de proteção NIST [11]

\begin{tabular}{c|ccc}
\hline Elemento & $\begin{array}{c}\text { Átomo } \\
\text { Excitado }\end{array}$ & $\begin{array}{c}\text { Comprimento } \\
\text { de Onda (nm) }\end{array}$ & $\begin{array}{c}\text { Intensidade } \\
\text { Relativa (\%) }\end{array}$ \\
\hline Argônio & Ar I & 912,2967 & 100,0 \\
Carbono & C I & 909,483 & 45,0 \\
& & 911,180 & 30,0 \\
Oxigênio & O I & 915,601 & 23,5 \\
\hline
\end{tabular}

A maior intensidade relativa da radiação emitida pelo Ar I resulta nos picos em torno de $914 \mathrm{~nm}$ : maior para a proteção de Argônio, intermediário para $\mathrm{Ar}+5 \% \mathrm{O}_{2} \mathrm{e}$, por fim, menor para $\mathrm{Ar}+8 \% \mathrm{CO}_{2}$. Para a adição de oxigênio, observa-se uma adição da radiação emitida pelo átomo excitado $\mathrm{O}$ I no pico existente de Ar I e, para a adição de dióxido de carbono observa-se dois picos distintos nas proximidades de $910 \mathrm{~nm}$. A diferença entre os valores dos comprimentos onde ocorrem os picos podem ser relacionados a problemas na calibração do espectrômetro.

Esses picos são proporcionais à porcentagem de átomos e, portanto, de átomos excitados de argônio presentes no arco. Acredita-se que essa proporcionalidade tenha uma relação direta com a condutividade elétrica de cada componente da composição. Segundo Scotti and Ponomarev [12] quanto maior a condutividade elétrica do elemento na temperatura da região do plasma, maior é a parcela de corrente, em percentual, que passa por estes. No caso do $\mathrm{Ar}+8 \% \mathrm{CO}_{2}$, por exemplo, espera-se que uma porcentagem maior que a variação de argônio (8\%) circule pelos átomos ionizados de $\mathrm{CO}_{2}$.

Segundo a distribuição de Planck, descrita por Incropera et al. [13], a magnitude da emissão aumenta com o aumento da temperatura. Portanto, além de possuir maior intensidade relativa de elementos ionizados, o teste com gás de proteção Argônio apresentou maior radiação de corpo negro, indicando maior temperatura na região medida pelo espectrômetro (arco, junta, e metal de adição).

Por fim, como mostra a Figura 12, para um mesmo nível de corrente, a soldagem pelo processo MIG/MAG apresentou maiores valores de radiação infravermelha quando comparado ao processo TIG.

Os resultados numéricos obtidos sobre a energia luminosa do arco (integral dos espectros) fornecem, portanto, a quantidade de energia mínima requerida para a sobreposição do arco por uma fonte de iluminação no infravermelho próximo. Neste caso, deve-se submeter esta fonte de iluminação à mesma metodologia experimental descrita neste trabalho e, comparando-se seus perfis em espectro e, principalmente a energia luminosa fornecida (Figura 4), é possível concluir se esta fonte é suficiente ou não para a obtenção de imagens do processo de soldagem sem a interferência da luminosidade do arco. 

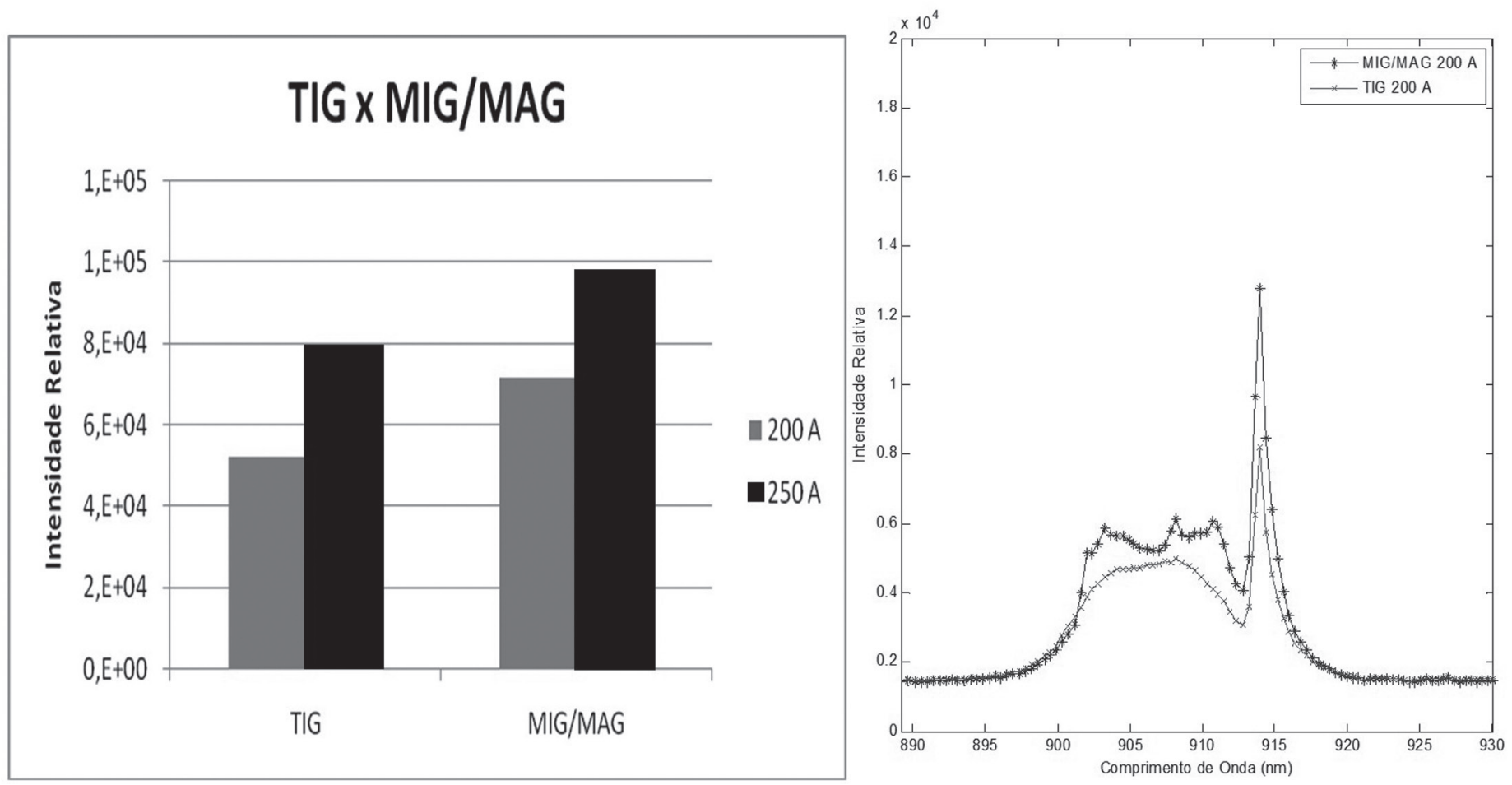

Figura 12. Resultados da Integração numérica para testes 3 a 6 (esquerda) e detalhe para forma dos espectros, com corrente de 200 A (direita).

\section{Conclusão}

Foi proposta, neste trabalho, a realização de um estudo da emissão e radiação infravermelha próxima em processos de soldagem a arco, em específico, em dois processos largamente utilizados, TIG e MIG/MAG, focando também sua influência por parâmetros como a corrente de soldagem e a proteção gasosa utilizada. Através do uso de um espectrômetro USB, aliado a um sistema de lentes para a focalização do arco e um algoritmo de aquisição do espectro, foi possível realizar um comparação quantitativa entre os processos e seus parâmetros.

Para ambos os processos, o aumento da intensidade de corrente resultou no aumento da emissão infravermelha. $\mathrm{O}$ aumento da tensão sobre um arco, de mesmo comprimento, aumenta a radiação de corpo negro no infravermelho e na emissão luminosa pelo retorno dos elétrons ao seu estado natural pelas partículas ionizadas. Para uma mesma intensidade de corrente, o processo MIG/MAG apresenta maior emissão e radiação infravermelha quando comparado ao processo TIG, devido à própria natureza física do arco.

$\mathrm{O}$ formato do espectro adquirido fornece informações importantes sobre o arco de soldagem. Seus picos indicam a composição da proteção gasosa utilizada e a temperatura atingida influencia na emissão de corpo negro. A soma dessas duas parcelas que caracteriza a emissão total do arco. Com base nos resultados obtidos é possível se obter quantitativamente a intensidade luminosa necessária para sobreposição do arco durante a aquisição de imagens.

\section{Agradecimentos}

Os autores agradecem a CAPES, pelo apoio financeiro com o fornecimento da bolsa de pós-graduação. Agradecem também ao grupo Laprosolda/UFU pelo espaço físico, apoio e auxílio no desenvolvimento deste trabalho. O Prof. Vilarinho gostaria de agradecer ao CNPq (Processo 473953/2009-9 e Processo 307554/2008-2) e à Fapemig (Processo TEC - APQ01389-08).

\section{Referências Bibliográficas}

[1] BÁLSAMO, P.S.S., et al. Development of an experimental technique for studying metal transfer in welding: synchronized shadowgraphy. Int. J. for the Joining of Materials v., p. 2000.

[2] ZHANG, Y.M., R. KOVACEVIC, and L. LI. Adaptive Control of Full Penetration Gas Tungsten Arc Welding. IEEE Transactions On Control Systems Technology, v.4, n. 4, p. 1996. [3] ZHANG, Y.M., L. LI, and R. KOVACEVIC, Monitoring of Weld Pool Appearance for Penetration Control, in Trends in Welding Research, Proceedings of 4th International Conference. 1995: Gatinburg, Tennessee. p. 4.

[4] BASKORO, A.S., et al., Welding Penetration Control for Aluminum Pipe Welding Using Omnidirectional Visonbased Monitoring of Molten Pool, in 8th Internation Welding Symposium. 2008: Kyoto International Conference Center, Kyoto, Japan. p. 1.

[5] VILARINHO, L.O., et al., Dedicated Near-Infrared Vision System for Monitoring Welding Processes, in 20th International 
Congress of Mechanical Engineering. 2009: Gramado, RS, Brasil. p. 7.

[6] HARA, K. and Y. SUGA, Penetration Control by Monitoring of Molten Pool Condition in TIG Welding of Thin Steel Metal, in 8th Internation Welding Symposium. 2008: Kyoto International Conference Center, Kyoto, Japan. p. 1.

[7] DUFFEY, T.P., S.E. SLOAN, and J. MAZUMDER, Measurement of Free Surface Deformation by Reflective Topography, in Trends in Welding Research, Proceedings of 4th International Conference. 1995: Gatinburg, Tennessee. p. 10.

[8] HOUGHTON, M.A., et al. Vision Systems for Monitoring and Control of Arc Welding Operations. Soldagem e Inspeção, v.12, n. 4, p. 283-299, 2007.

[9] CHENEY, W. and D. KINCAID. Numerical Mathematics and Computing. Sixth Edition ed. Belmont, 2007. 763 p. p.

[10] VILARINHO, L.O. O arco TIG a 100 A e argônio puro é um arco especial? Soldagem e Inspeção, v.14, n. 4, p. 353-357, 2009.

[11] NIST Atomic Spectra Database Lines Data (version 3.1.5). 2010, NIST.

[12] SCOTTI, A. and V. PONOMAREV. Soldagem MIG/MAG: melhor entendimento, melhor desempenho. São Paulo, 2008. $284 \mathrm{p}$.

[13] INCROPERA, et al. Fundamentals os Heat and Mass Transfer. Sixth Edition ed, 2002. 999 p. 